\title{
Human ether-à-go-go-related gene expression is essential for cisplatin to induce apoptosis in human gastric cancer
}

\author{
RIPING ZHANG ${ }^{1 *}$, PEI TIAN ${ }^{2 *}$, QIANG CHI $^{1}$, JIZHOU WANG $^{3}$, YONGWEI WANG $^{3}$, \\ LINGYU SUN ${ }^{4}$, YANG LIU ${ }^{1}$, SULI TIAN ${ }^{4}$ and QIFAN ZHANG ${ }^{4}$ \\ ${ }^{1}$ Department of General Surgery, The Second Affiliated Hospital of Harbin Medical University; \\ ${ }^{2}$ Department of Ophthalmology, The First Affiliated Hospital of Harbin Medical University; ${ }^{3}$ Department \\ of General Surgery, The First Affiliated Hospital of Harbin Medical University; ${ }^{4}$ Center of Cancer \\ Research, The Fourth Affiliated Hospital of Harbin Medical University, Harbin, P.R. China
}

Received August 8, 2011; Accepted September 28, 2011

DOI: 10.3892/or.2011.1515

\begin{abstract}
Human ether-à-go-go-related gene (HERG) is overexpressed in a wide range of human cancers and regulates survival, migration, and apoptosis. The aim of this study was to investigate the role of HERG in cisplatin-induced apoptosis in gastric cancer in vitro and in vivo. siRNA was used to silence HERG expression. HERG expression was detected by Western blot analysis in vitro, and further confirmed by immunohistochemistry in vivo. Chemosensitivity to cisplatin in gastric cancer cells was analyzed with a Cell Counting Kit-8 assay. Apoptosis was detected by flow cytometry in vitro, and in situ apoptotic SGC7901 human gastric tumor cells in BALB/c nude mice were detected by terminal deoxynucleotidyl transferase dUTP nick-end labeling. Our results show that cisplatin increased the expression of HERG in gastric cancer cells. Silencing HERG inhibited the apoptosis induced by cisplatin both in vitro and in vivo, by attenuating the cisplatin effects on Bcl-2, Bax and active caspase-3. The role of HERG in modulation of cisplatin-induced apoptosis suggests that HERG may provide a new potential target for cisplatin chemotherapy in human gastric cancer.
\end{abstract}

\section{Introduction}

Gastric cancer is one of the most common malignancies worldwide. A total of 989,600 new cases and 738,000 deaths were estimated to have occurred in 2008, accounting for $8 \%$ of the

Correspondence to: Dr Q. Zhang or Dr S. Tian, Center of Cancer Research, The Fourth Affiliated Hospital of Harbin Medical University, Harbin, P.R. China

E-mail: zhangqifan2000@hotmail.com

E-mail: tiansuli1954@126.com

${ }^{*}$ Contributed equally

Key words: human ether-à-go-go-related gene, cisplatin, chemosensitivity, apoptosis, gastric cancer total cancer cases and $10 \%$ of total cancer deaths (1). Most patients are either diagnosed at an advanced stage, or in relapse after surgery, and systemic chemotherapy is the only treatment option available in this situation. The platinum-based drug cisplatin is widely used to treat many solid tumors including gastric cancers (2). Like most chemotherapeutic drugs, cisplatin exerts its anti-cancer effect by inducing apoptosis, probably mediate through caspase-3 activity (3). Cytotoxicity of cisplatin, and its ability to induce cancer cell apoptosis is influenced by modulators of potassium ion flux (4).

Potassium channels are the most diverse class of plasma membrane ion channels, and they participate in many and diverse physiological events such as excitability, contraction, cell cycle progression and metabolism in different cell types (5-7). One of the potassium channels most commonly related to cell proliferation and cancer is the human ether-à-go-go-related gene (HERG) potassium channel $(8,9)$. HERG potassium channel is mainly expressed in the cell membrane of cardiac myocytes, endocrine cells and lymphocytes, and genetic mutation in HERG can result in long QT syndrome, a lethal cardiac arrhythmia (10). HERG is overexpressed in a wide range of human cancers, such as gastric cancer, colorectal cancer, endometrial cancer and glioblastoma multiforme (11-14). Three main functions relevant to tumor cell biology can be ascribed to HERG activity: tumor cell proliferation (15), tumor cell invasiveness (12) and tumor neoangiogenesis (16). Because of their oncogenic properties, distribution, modulation and pharmacology, HERG has gained great interest as potential diagnostic markers and membrane therapeutic targets for cancer $(17,18)$.

HERG is specifically overexpressed in human gastric cancer, whereas it is not expressed in the normal gastric mucosa epithelium, and the expression is associated with the differentiation and clinical staging of gastric cancer $(11,19,20)$. However, little is known about the correlation between HERG expression and the mechanism by which cisplatin inhibits gastric cancer cell growth. Therefore, this study investigates the possible alteration of HERG expression in cisplatin-treated gastric cancer cells, the relationship between HERG expression and chemosenitivity to cisplatin in gastric cancer cells, and the role of HERG in cisplatin-induced apoptosis. 


\section{Materials and methods}

Mice, cells and reagents. Male nude BALB/c mice, 8 weeks-old, were obtained from the Animal Research Center, the Second Affiliated Hospital of Harbin Medical University, China. The human gastric cancer cell lines SGC7901 and MGC803 were obtained from the Institute of Biochemistry and Cell Biology, Chinese Academy of Sciences, Shanghai, China. The cells were cultured at $37^{\circ} \mathrm{C}$ in RPMI-1640 medium (Gibco BRL, Grand Island, NY, USA) supplemented with $10 \%$ fetal calf serum (FCS). Antibodies against HERG, Bcl-2, Bax, caspase-3 and GAPDH (Santa Cruz Biotechnology, CA, USA) were used. Cisplatin was purchased from Sigma (St. Louis, MO, USA).

Transfection of siRNA targeting the HERG. The control siRNA and HERG siRNA were purchased from Santa Cruz Biotechnology. SGC7901 and MGC803 cells were grown to $50 \%$ confluence in 6- or 96-well plates, and transfected with the siRNAs in serum-free medium without antibiotic supplements using Lipofectamine ${ }^{\mathrm{TM}} 2000$ (Invitrogen, CA, USA). The cells were incubated for $4-6 \mathrm{~h}$, then the culture media were refreshed, and the cells were cultured for a further $48 \mathrm{~h}$. Silencing of HERG expression was confirmed by Western blot analysis.

CCK8 assay. SGC7901 and MGC803 cells were seeded into a 96-well plate ( $3 \times 10^{3} /$ well), and cultured overnight. The culture medium was replaced with fresh serum-free medium or the same medium containing control siRNA or HERG siRNA, and the cells were cultured for 4-6 h. The medium was again replaced with $200 \mu \mathrm{l}$ of fresh RPMI-1640 medium, or the same medium containing cisplatin at various concentrations. The cells were cultured for $48 \mathrm{~h}$, and cell viability was measured with a Cell Counting Kit-8 (CCK-8) kit (Dojindo Molecular Technologies, Gaithersburg, MD, USA). The proliferation inhibition rate (in percentage) was calculated according to the following formula: (1 - experimental OD value/control OD value) $\mathrm{x} 100 \%$. The experiments were repeated thrice.

Apoptosis assay. Control siRNA- or HERG siRNA-transfected SGC7901 and MGC803 cells were incubated with or without cisplatin $(0.8 \mathrm{mg} / 1$ for SGC7901 and $0.4 \mathrm{mg} / \mathrm{l}$ for MGC803) as described above for $48 \mathrm{~h}$. Cells $\left(1 \times 10^{5}\right)$ were harvested, washed with PBS, resuspended in $100 \mu$ l of binding buffer, and incubated with $5 \mu \mathrm{l}$ of Annexin $\mathrm{V}$ and $5 \mu \mathrm{l}$ of PI for $15 \mathrm{~min}$ according to the manufacturer's instruction (BD Biosciences, San Jose, CA, USA). The cells were analyzed in a cytometer (Beckman Coulter, CA, USA) to measure the apoptosis rate. The cells that had undergone apoptosis were viewed by laser scanning confocal microscopy (LSM-510, Carl Zeiss Jena GmbH, Jena, Germany).

Animal model and treatments. All surgical procedures and care administered to the animals were in accordance with institutional guidelines, and have been described previously (21). SGC7901 cells $\left(5 \times 10^{6}\right)$ were subcutaneously injected into the back of the mice. Tumor volumes were estimated according to the following formula: $\pi / 6 \mathrm{x} \mathrm{a}^{2} \mathrm{xb}$, where $\mathrm{a}$ is the short axis, and $\mathrm{b}$ the long axis. When tumors reached around $100 \mathrm{~mm}^{3}$, the mice were randomly assigned to four groups (each group had 18 mice): control siRNA, HERG siRNA, cisplatin + control siRNA and cisplatin + HERG siRNA. Equal volumes of $20 \mathrm{nM}$ siRNA and Lipofectamine 2000 were mixed together, and the mixture was further mixed with an equal volume of serum-free RPMI-1640 medium. To standardize the experiments, mice in each group received both intratumoral and i.p. injections. In the control siRNA and HERG siRNA groups, mice received i.p. injection of $200 \mu \mathrm{l}$ of PBS weekly, and intratumoral injection of $250 \mathrm{pmol}$ of control siRNA and HERG siRNA, respectively, in a $50 \mu \mathrm{l}$ siRNA transfection solution weekly. In the cisplatin + control siRNA and cisplatin + HERG siRNA groups, mice received i.p. injection of $200 \mu \mathrm{l}$ of cisplatin (diluted in PBS) at the dose of $7 \mathrm{mg} / \mathrm{kg}$ weekly and intratumoral injection of control siRNA and HERG siRNA, respectively, at the same weekly dose as above. Six mice from each group were randomly sacrificed 1 and 2 weeks after treatment started, and their tumors were excised. The remaining mice were monitored for 3 weeks.

Immunohistochemistry. Tumor sections $(4 \mu \mathrm{m})$ were blocked with 3\% BSA, incubated with primary Abs, and subsequently incubated with secondary Abs using the Ultra Sensitive TMS-P kit (Zhongshan, Beijing, China). Immunoreactivity was developed with Sigma FAST DAB (3,3'-diaminobenzidine tetrahydrochloride) and $\mathrm{CoCl}_{2}$ enhancer tablets (Sigma-Aldrich, Shanghai, China). Sections were counterstained with hematoxylin, mounted and examined under a microscope.

In situ detection of apoptotic cells. This method has been described previously (22). In brief, tumor sections were stained with terminal deoxynucleotidyl transferase dUTP nick-end labeling (TUNEL) (Roche, Shanghai, China). The total number of apoptotic cells in 10 randomly selected high-power (x400) fields under a microscope was counted. The apoptosis index was calculated according to the formula: number of apoptotic cells/total number of nucleated cells x $100 \%$.

Western blot analysis. This method has been described previously (23). In brief, cells or tumor tissues were homogenized in protein lysate buffer. Debris was removed by centrifugation at $12,000 \mathrm{xg}$ for $10 \mathrm{~min}$ at $4^{\circ} \mathrm{C}$. The protein content of the cell and tumor tissue homogenates was determined, and protein samples were resolved on $12 \%$ polyacrylamide SDS gels, and electrophoretically transferred to polyvinylidene difluoride membranes. The membranes were blocked with $3 \%$ BSA, incubated with primary antibodies, and subsequently with an alkaline phosphatase conjugated secondary antibodies. They were visualized with 5-bromo-4-chloro-3-indolyl phosphate/ nitro blue tetrazolium (Tiangen Biotech, Beijing, China).

Statistical analysis. The half maximal inhibitory concentration $\left(\mathrm{IC}_{50}\right)$ was calculated with a logit method. All other data were expressed as mean values \pm standard deviation (SD). One-way analysis of variance (ANOVA) and Student's t-test were used to evaluate statistical significance. $\mathrm{p}<0.05$ was set as statistical significance.

\section{Results}

Effects of HERG siRNA and cisplatin on HERG expression in gastric cancer cells. SGC7901 and MGC803 cells were treated with HERG siRNA or different concentrations of cisplatin 
A
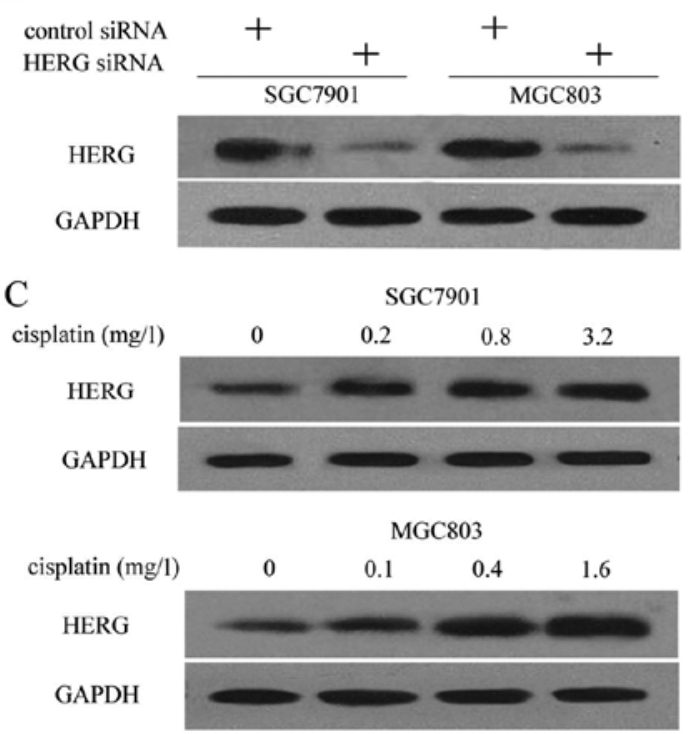

D

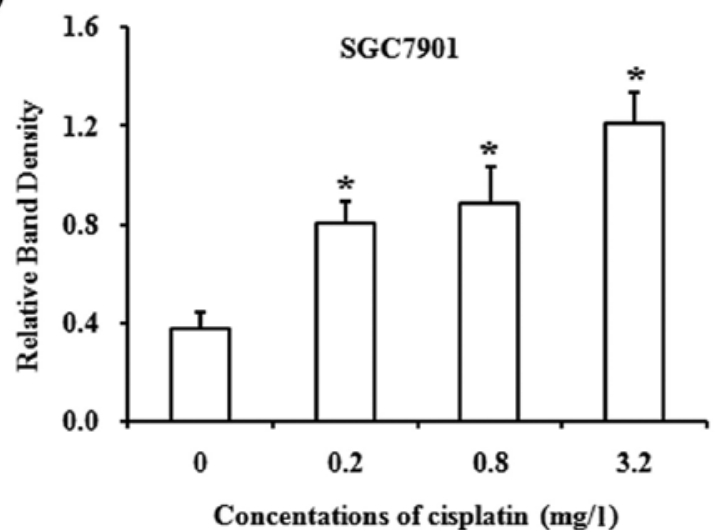

B

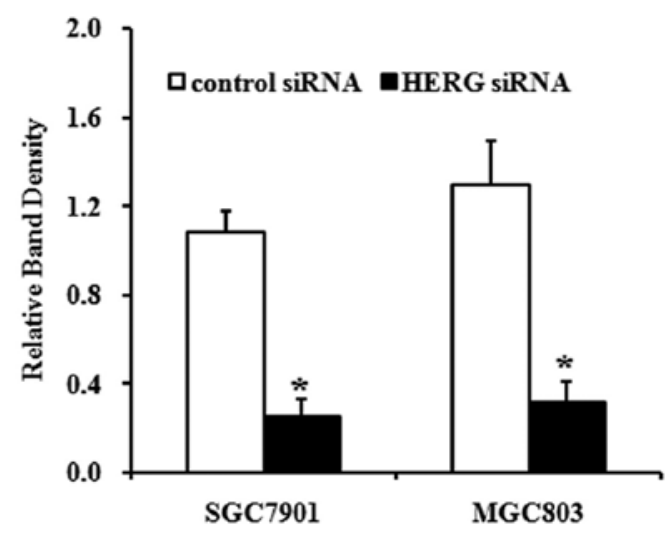

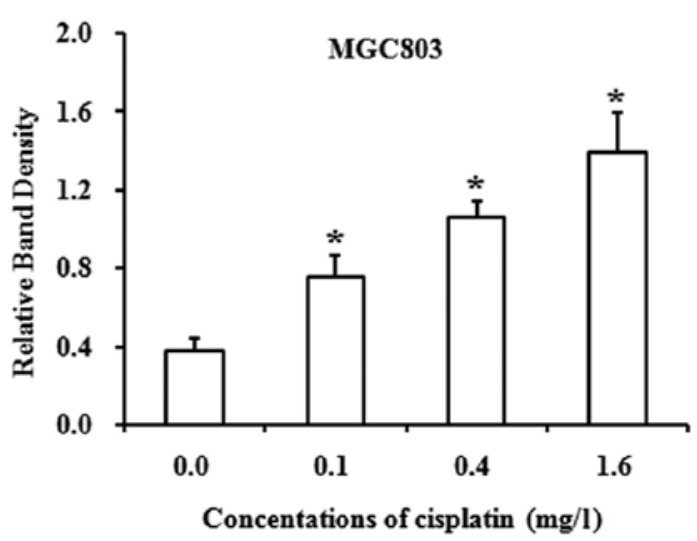

Figure 1. SGC7901 and MGC803 cells were incubated with HERG siRNA (A) or different concentrations of cisplatin (C) for 48 h, and cells were lysed and subjected to Western blot analysis to detect expression of HERG. (B and D) The density of each band (from A and C, respectively) was measured and compared with that of the internal control, GAPDH. *Significant difference in band density from the control.

A

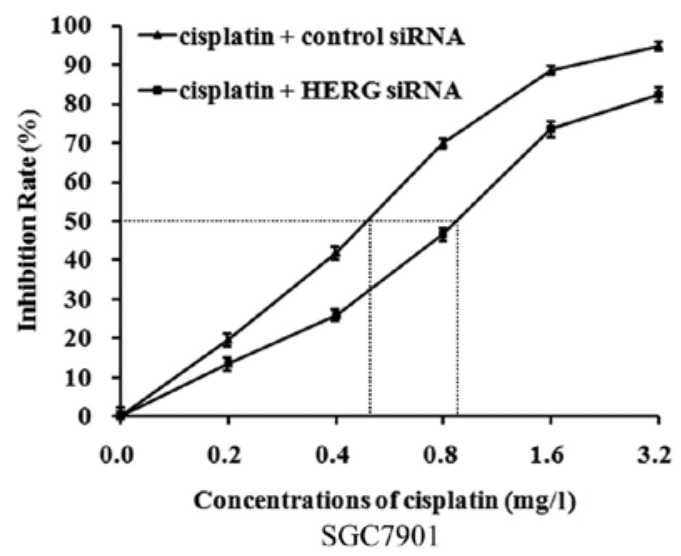

B

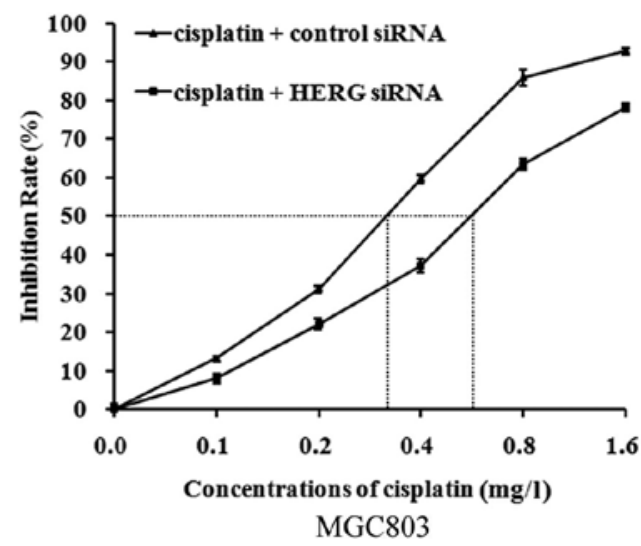

Figure 2. SGC7901 (A) and MGC803 (B) cells transfected with control siRNA or HERG siRNA were incubated with different concentrations of cisplatin. Forty-eight hours later, the proliferation of cells was assessed by the CCK8 method to calculate the proliferation inhibition rate. The dotted lines show the concentration of cisplatin, which resulted in $50 \%$ of maximal proliferation inhibition $\left(\mathrm{IC}_{50}\right)$ of cells.

for $48 \mathrm{~h}$. Expression of HERG was evaluated by Western blot analysis. As shown in Fig. 1A and B, transfection of cells with
HERG siRNA significantly reduced the expression of HERG in SGC7901 and MGC803 cells, compared with the cells trans- 

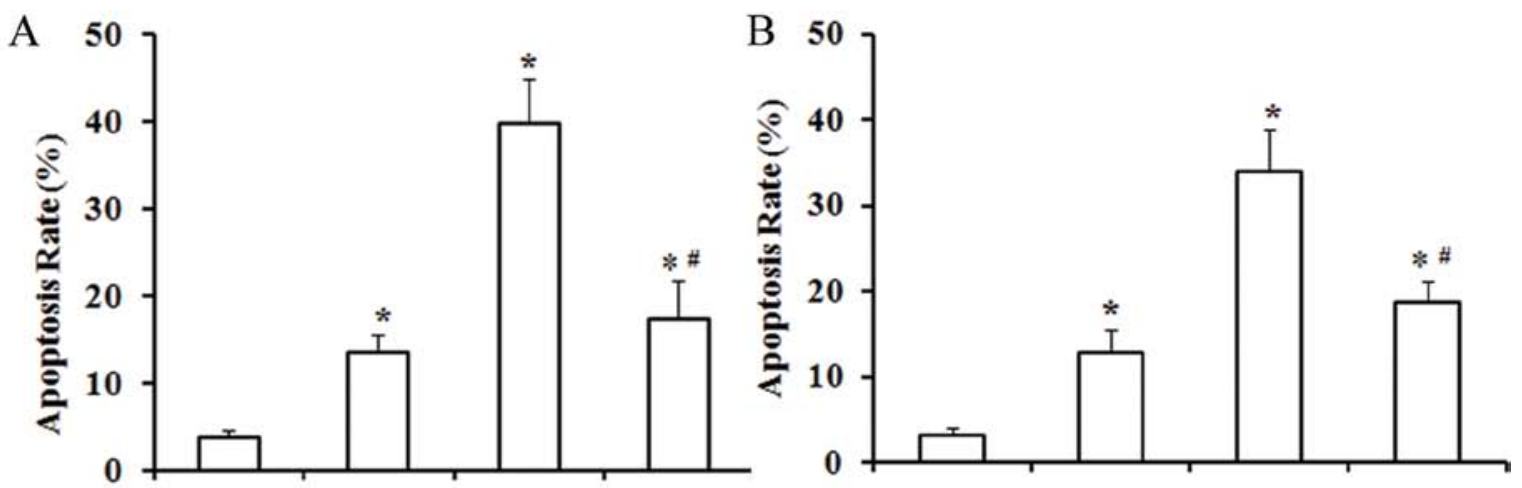

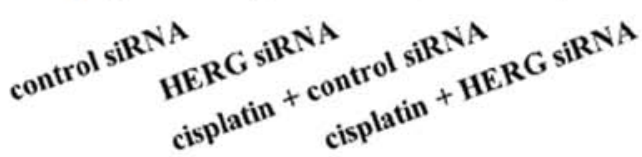

SGC7901

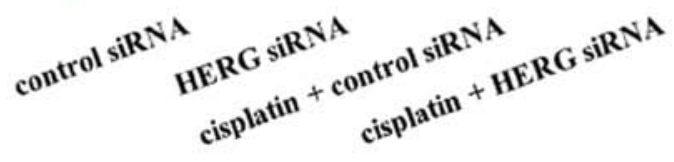

MGC803

C
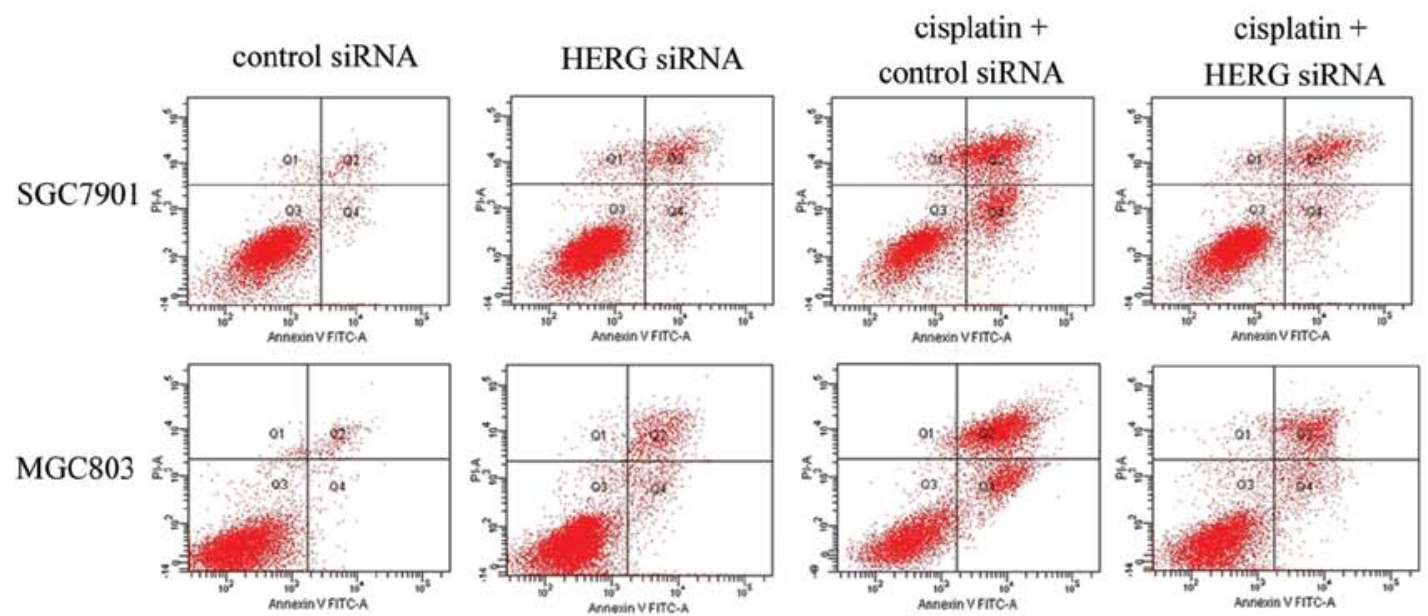

D

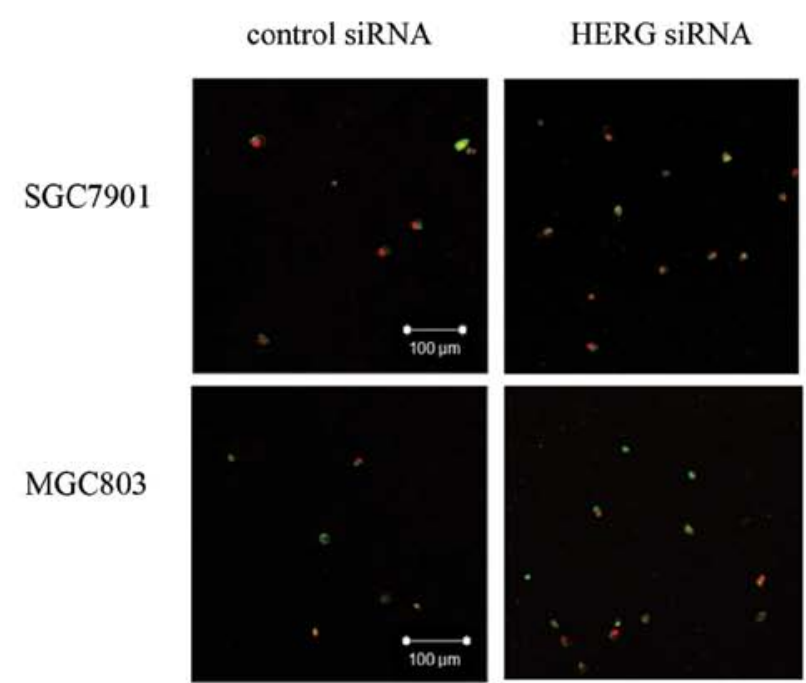

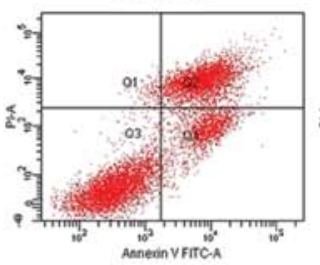

cisplatin +

control siRNA
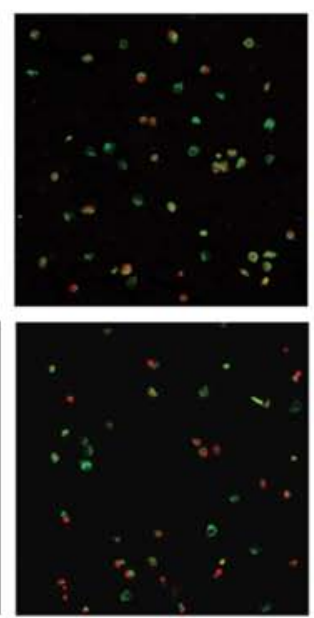

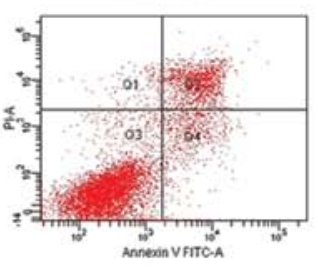

cisplatin +

HERG SiRNA
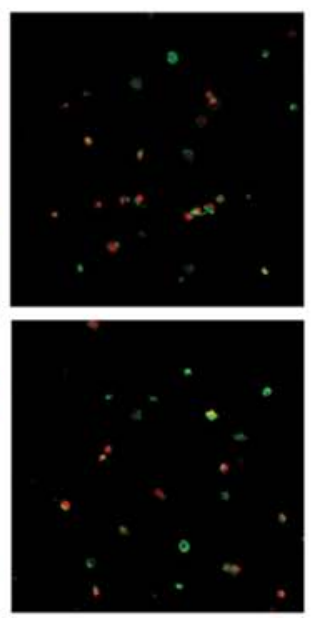

Figure 3. SGC7901 (A) and MGC803 (B) cells transfected with control siRNA or HERG siRNA were incubated with or without cisplatin. Forty-eight hours later, cells were harvested and flow cytometry was performed to measure apoptosis rates. "Significant increase in the apoptosis rate from control; " $\mathrm{Significant}$ increase compared with cisplatin-treated cells. (C) Representative histograms from cytometrically analyzed SGC7901 (upper panel) and MGC803 (lower panel) cells treated with control siRNA, HERG siRNA, cisplatin + control siRNA or cisplatin + HERG siRNA. (D) Cells were viewed by laser scanning confocal microscopy. Representative photographs were taken of SGC7901 (upper panel) and MGC803 (lower panel) cells treated with control siRNA, HERG siRNA, cisplatin + control siRNA or cisplatin + HERG siRNA.

fected with control siRNA ( $\mathrm{p}<0.001)$. Expression of HERG in SGC7901 and MGC803 cells was significantly upregulated after incubation with different concentrations of cisplatin $(\mathrm{p}<0.01)$, as shown in Fig. 1C and D). 
A
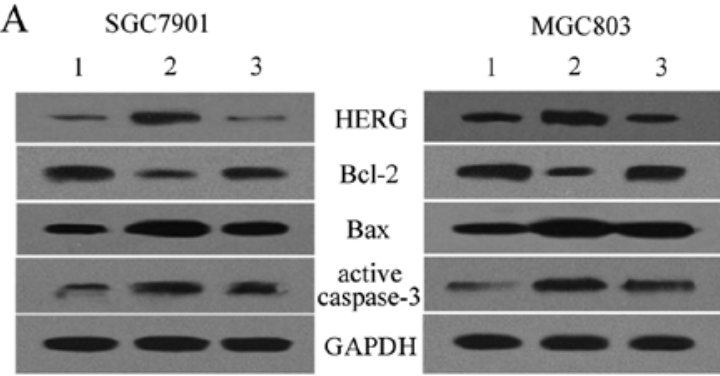

B
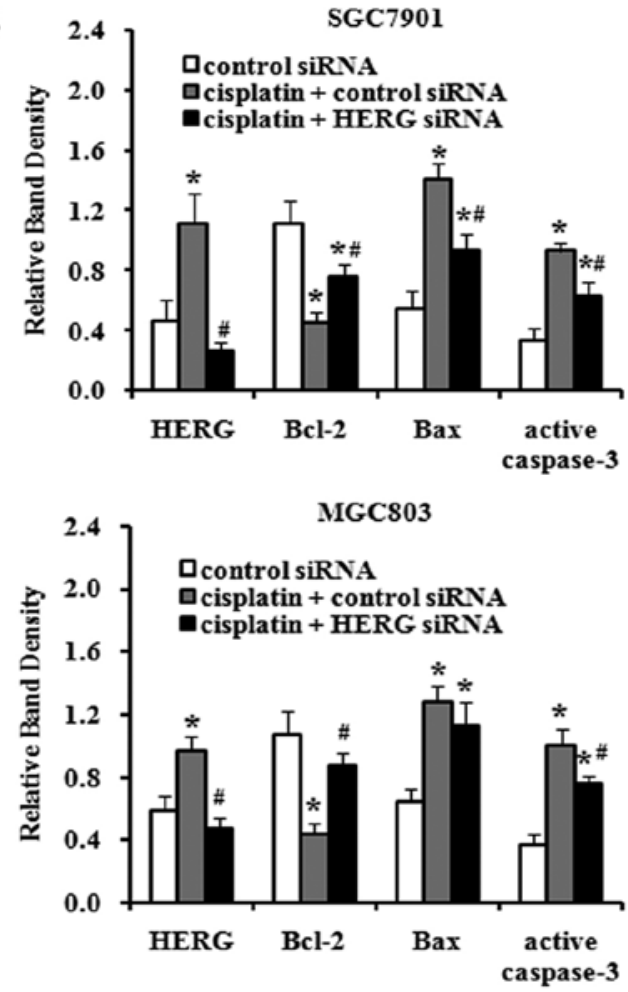

Figure 4. (A) SGC7901 and MGC803 cells were treated with control siRNA (lane 1), cisplatin + control siRNA (lane 2), or cisplatin + HERG siRNA (lane 3) for $48 \mathrm{~h}$. Homogenates of the cells were subjected to Western blot analysis to detect the expression of HERG, Bcl-2, Bax and active caspase-3. (B) The density of each band was measured and compared with that of the interna control, GAPDH. "Significant difference in band density from control siRNA group. "Significant difference from cisplatin + control siRNA group.

HERG SiRNA reduces the chemosensitivity of gastric cancer cells to cisplatin. SGC7901 and MGC803 cells transfected with control siRNA or HERG siRNA were incubated with different concentrations of cisplatin for $48 \mathrm{~h}$, and $\mathrm{IC}_{50}$ values of cells were determined with a CCK8 assay. With a logit method (24), the $\mathrm{IC}_{50}$ of cisplatin in SGC7901 cells transfected with control siRNA or HERG siRNA was 0.47 or $0.87 \mathrm{mg} / \mathrm{l}$, respectively (Fig. 2A). Similar results were obtained with MGC803 cells, whose $\mathrm{IC}_{50}$ of cisplatin was 0.31 or $0.58 \mathrm{mg} / 1$, respectively (Fig. 2B). In terms of $\mathrm{IC}_{50}$ value, SGC7901 and MGC803 cells transfected with HERG siRNA were less sensitive to cisplatin than those transfected with control siRNA.

HERG siRNA reduces cisplatin-induced apoptosis of gastric cancer cells. SGC7901 and MGC803 cells transfected with control siRNA or HERG siRNA were incubated with or without cisplatin $(0.8 \mathrm{mg} / 1$ for SGC7901 and $0.4 \mathrm{mg} / \mathrm{l}$ for
MGC803) for $48 \mathrm{~h}$. The cells were subjected to flow cytometry to measure the rates of apoptosis, and viewed by laser scanning confocal microscopy. As shown in Fig. 3A, both HERG siRNA and cisplatin + control siRNA increased the apoptosis rate of SGC7901 cells $(13.6 \pm 2.1 \%$ and $39.7 \pm 5.1 \%$, respectively) compared with the control $(3.8 \pm 1.0 \%)$ (both $\mathrm{p}<0.05)$, whereas cisplatin + HERG siRNA induced a lower apoptosis rate $(17.4 \pm 4.5 \%)$ compared with that induced by cisplatin + control siRNA $(\mathrm{p}<0.001)$. Similar results were obtained with MGC803 cells (Fig. 3B). Laser scanning confocal microscopy confirmed that HERG siRNA reduced cisplatin-induced apoptosis, as shown in the representative photographs (Fig. 3D). SGC7901 and MGC803 cells treated with cisplatin + HERG siRNA experienced less early- and late-staged apoptotic cells, than cells treated with cisplatin + control siRNA.

HERG siRNA attenuates the effects of cisplatin on apoptosisrelated proteins in gastric cancer cells. SGC7901 and MGC803 cells were treated as above for $48 \mathrm{~h}$, and apoptosis-related proteins, including $\mathrm{Bcl}-2$, Bax and active caspase-3, were detected by Western blot analysis. As shown in Fig. 4A, cisplatin in combination with either control siRNA or HERG siRNA upregulated the expression of Bax and active caspase-3, and downregulated Bcl-2 expression in SGC7901 cells, but the effects were weaker in the HERG siRNA cells. Similar changes in the three proteins were observed in MGC803 cells. Rather, the expression of Bax was slightly downregulated in MGC803 cells treated with cisplatin + HERG siRNA compared with cisplatin + control siRNA, though this difference did not reach significance after quantitative analysis (Fig. 4B).

HERG siRNA reduces the tumoral cell apoptosis induced by cisplatin in mice. As shown in Fig. 5A, i.p. injection of cisplatin upregulated the expression of HERG in subcutaneous SGC7901 tumors, compared with control, whereas intratumoral injection of HERG siRNA attenuated the upregulation of HERG induced by cisplatin.

Tumor sections were stained with TUNEL to detect in situ apoptotic cells. The apoptotic cells were counted to determine the apoptosis index. As shown in Fig. 5C, HERG siRNA increased the apoptosis index 1 -fold $(\mathrm{p}<0.05)$, and cisplatin + control siRNA increased the apoptosis index 5-fold ( $\mathrm{p}<0.001)$, compared with the control. However, the apoptosis index of tumors treated with cisplatin + HERG siRNA was only 3-fold higher $(\mathrm{p}<0.001)$ than that of the control group and was significantly lower than the ciplatin + control siRNA group $(\mathrm{p}<0.001)$.

Bcl-2, Bax and active caspase- 3 were also detected by Western blot analysis of tumor homogenates (Fig. 5D). Cisplatin + control siRNA significantly upregulated the expression of Bax and active caspase-3, and downregulated Bcl-2 expression, while cisplatin + HERG siRNA had weaker effects (Fig. 5E), in accordance with our in vitro findings.

Next, we examined the effects of cisplatin in combination with control siRNA or HERG siRNA on the growth of subcutaneous SGC7901 tumors that received treatments of control siRNA, HERG siRNA, cisplatin + control siRNA, or cisplatin + HERG siRNA. As shown in Fig. 5F, the tumors treated with control siRNA grew remarkably fast, reaching $2100 \pm 200 \mathrm{~mm}^{3}$ after 3 weeks. In contrast, tumors treated with HERG siRNA or cisplatin + control siRNA were significantly (both $\mathrm{p}<0.05)$ 
A

control siRNA

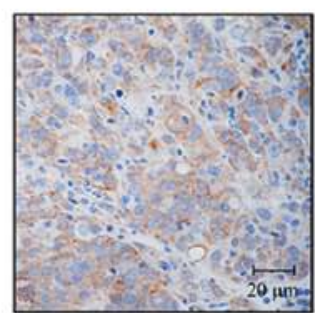

B

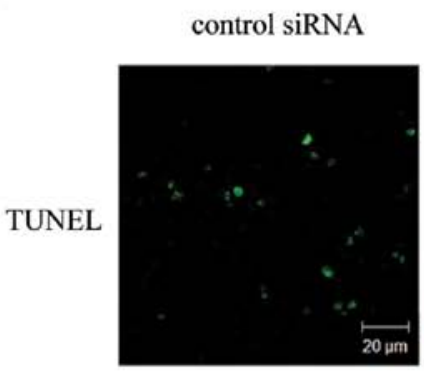

$\mathrm{C}$

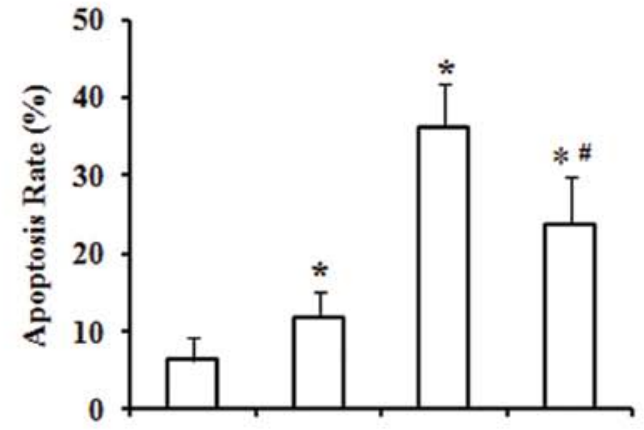

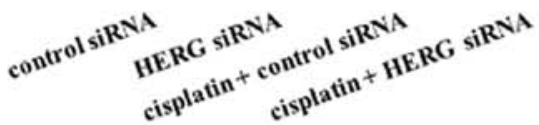

cisplatin +

control siRNA

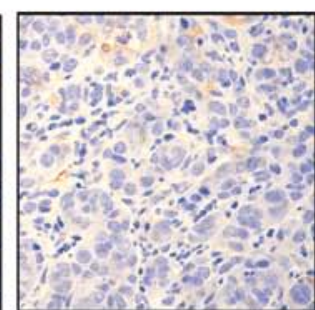

HERG SiRNA

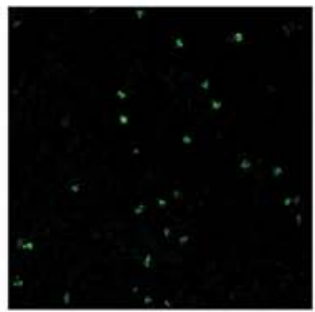

$\mathrm{D}$

cisplatin + control siRNA
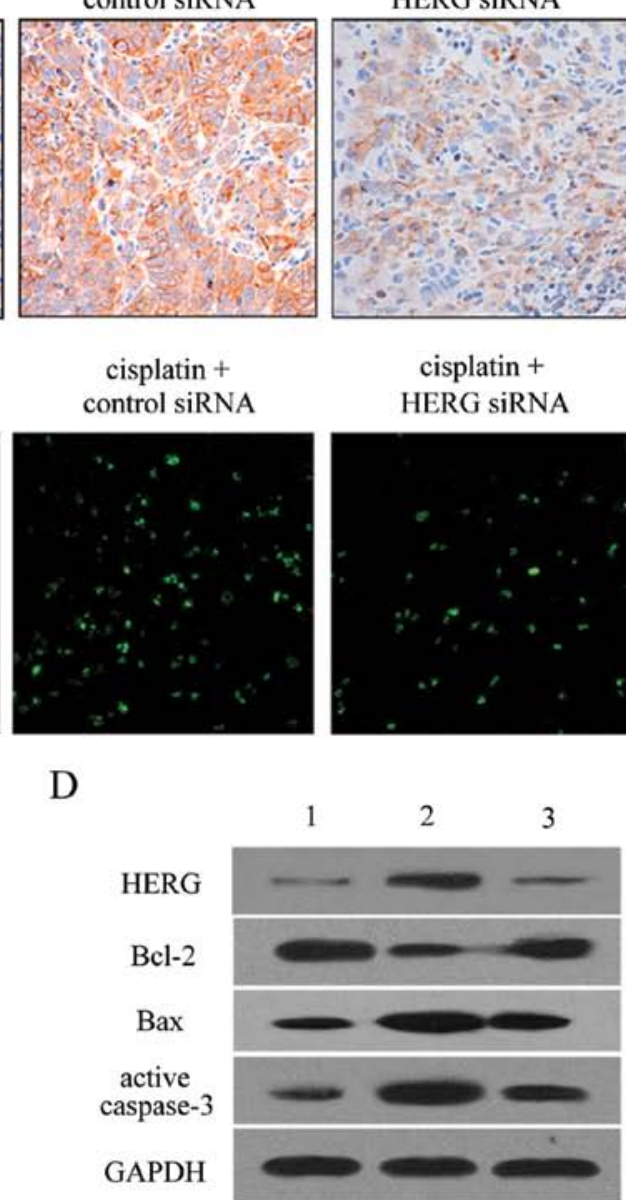

$\mathrm{E}$

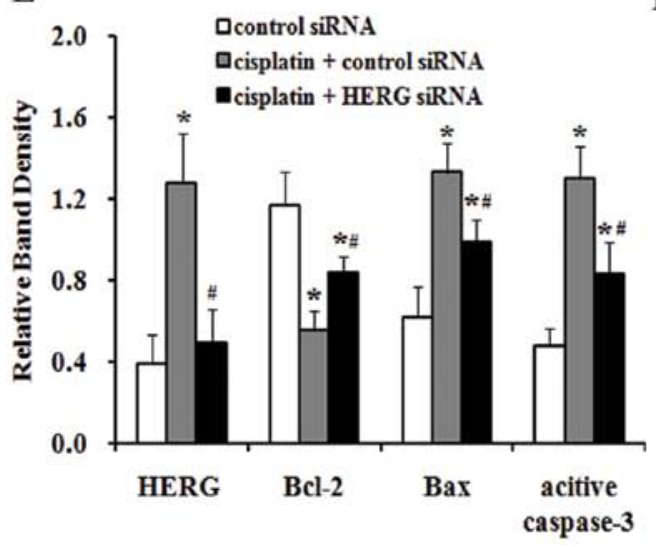

$\mathrm{F}$

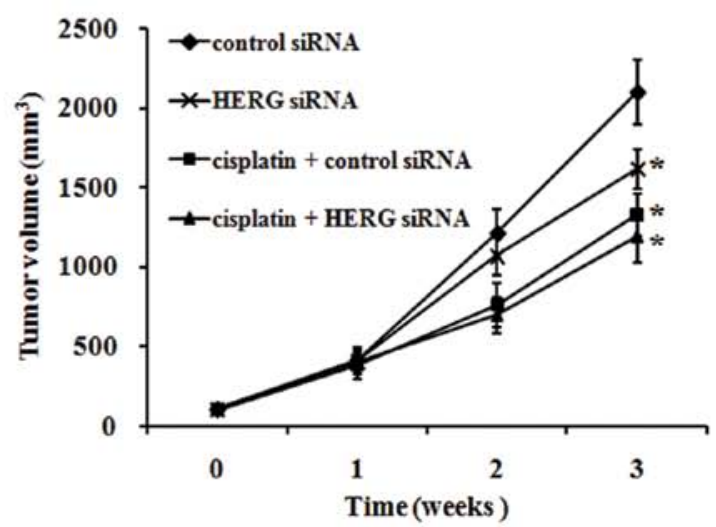

Figure 5. Mice were subcutaneously injected with SGC7901 cells. When tumors reached around $100 \mathrm{~mm}^{3}$ in volume, mice received treatment with control siRNA, HERG siRNA, cisplatin + control siRNA, or cisplatin + HERG siRNA. (A) HERG expression in subcutaneous SGC7901 tumors was detected by immunohistochemical analyses. Sections were prepared from tumors 1 week after receiving treatment. (B) Tumoral cell apoptosis was detected by TUNEL. Illustrated are representative sections prepared from subcutaneous tumors 2 weeks after treatment. (C) TUNEL-positive cells were counted to give the apoptosis index. (D) Two weeks after treatment, tumors from control mice (lane 1), cisplatin + control siRNA (lane 2), and cisplatin + HERG siRNA (lane 3) were homogenized and subjected to Western blot analysis to measure the expression of HERG, Bcl-2, Bax and active caspase-3. (E) The density of each band from (D) was measured and compared to that of the internal control, GAPDH. (F) The sizes $\left(\mathrm{mm}^{3}\right)$ of tumors were monitored and recorded for 3 weeks. "Significant difference from control siRNA group.

smaller, reaching only $1617 \pm 123$ and $1327 \pm 165 \mathrm{~mm}^{3}$ in volume, respectively, compared with control siRNA treated tumors.
However, tumors treated with cisplatin + HERG siRNA were $1189+165 \mathrm{~mm}^{3}$, which were not significantly different from 
tumors treated with cisplatin + control siRNA. We calculated the value of CDI to investigate whether the effects of HERG siRNA and cisplatin are additive or antagonistic as described previously (21). A value of CDI less than, equal to or greater than 1 indicates that the drugs are synergistic, additive or antagonistic, respectively. The CDI value for HERG siRNA and cisplatin was 1.2 , which was $>1$, indicating the two agents had antagonistic effect in inhibiting tumor growth.

\section{Discussion}

We have, for the first time, demonstrated that HERG expression is significantly upregulated when the gastric cancer cells SGC7901 and MGC803 are treated with cisplatin. Silencing of HERG expression reduced the chemosensitivity of gastric cancer cells to cisplatin and inhibited cisplatin-induced apoptosis, which indicates that HERG expression is essential for cisplatin to induce apoptosis in human gastric cancer. These results were confirmed both in vitro and in vivo.

Recent studies have provided substantial evidence that potassium channels are involved in the regulation of tumor cell proliferation, acquirement of invasive, metastatic ability, apoptosis, cell cycle and even drug resistance $(4,6,25,26)$. Among these potassium channels, HERG has demonstrated the strongest relationship with human cancer, and been considered as a potential therapeutic target $(25,27)$. Shao et al found that HERG was exclusively expressed in gastric cancer and that the expression of HERG was associated with tumor differentiation, TNM stage and lymph node involvement in gastric cancer (11). However, there are no reports on the alteration of HERG expression after treatment with chemotherapeutic anti-cancer drugs. In the present study, we found that after exposure to cisplatin, HERG expression significantly increased, indicating that this may be one of the mechanisms accounting for the reaction of gastric cancer to cisplatin.

One other study has investigated the potential relationship between HERG expression and the chemosensitivity of gastric cancer cells to cisplatin. Chen et al (28) reported that A549 cells expressing low levels of HERG were less sensitive than HT-29 cells expressing high levels of HERG to vincristine, paclitaxel and hydroxy-camptothecin. The chemosensitivities of HERG-transfected A549 cells to vincristine, paclitaxel and hydroxy-camptothecin were significantly increased, compared to parent A549 cells. Here we established that the cisplatin $\mathrm{IC}_{50}$ of gastric cancer cells treated with cisplatin alone was much lower than that of cells treated with the combination of cisplatin and HERG siRNA. This result indicates that siRNA-mediated HERG silencing reduces the chemosensitivity of gastric cancer cells to cisplatin. These findings lead us to presume that HERG plays a positive role in the reaction to cisplatin in the gastric cancer cells.

Apoptosis is the process by which most chemotherapeutic anti-cancer drugs induce cell death. Like most chemotherapeutic drugs, cisplatin exert its tumor killing action by inducing apoptosis (3). Our flow cytometry results demonstrated that less apoptotic cells were detected when the gastric cancer cells were incubated with cisplatin + HERG siRNA than when they were incubated with cisplatin + control siRNA. Detection of apoptosis-related proteins (Bcl-2, Bax and active caspase-3) showed that in both cell lines HERG siRNA attenuates the effect of cisplatin on apoptosis-related proteins. Bcl-2, which is located on the mitochondrial membrane, stabilizes the mitochondrial membrane potential and prevents the release of apoptosis-inducing molecules such as cytochrome c. Therefore, we presumed that HERG siRNA inhibits cisplatin-induced apoptosis through the intrinsic pathway mediated by members of the Bcl-2 family.

In vivo analyses demonstrated that i.p. injection of cisplatin upregulated the expression of HERG in subcutaneous SGC7901 tumors in mice, and intratumoral injection of HERG siRNA inhibited HERG expression. The in situ apoptosis rate of tumors treated with the combination of cisplatin + HERG siRNA was significantly lower than that of cisplatin + control siRNA, which is in full accord with the in vitro study. Both HERG siRNA and cisplatin could inhibit the subcutaneous tumor growth alone. However, the combination of HERG siRNA and cisplatin showed no significant difference from cisplatin alone. The CDI value indicated the antagonistic effect of HERG siRNA and cisplatin in vivo. We supposed that the antagonistic effect was, at least in part, attributed to the reduction of tumoral cell apoptosis in cisplatin + HERG siRNA group.

Wang et al (29) found that HERG is a regulator of tumor cell apoptosis and proliferation, and that $\mathrm{H}_{2} \mathrm{O}_{2}$ can induce considerable apoptosis in cells expressing endogenous or cloned HERG. When the HERG was blocked, $\mathrm{H}_{2} \mathrm{O}_{2}$-induced apoptosis was also inhibited. Han et al (30) reached a similar conclusion that HERG facilitates cellular DNA fragmentation in HEK cells via concomitant activation of MAP kinases and inactivation of Akt. In our study, when the gastric cancer cells were treated with cisplatin, HERG expression increased in both cell lines and silencing HERG by siRNA inhibited the apoptosis induced by cisiplatin, indicating that HERG expression may be essential for cisplatin to induce apoptosis in human gastric cancer.

From the phenomena discovered in our study, we conclude that HERG is an important factor that regulates apoptosis in gastric cancer cells and that the cisplatin induction of apoptosis in gastric cancer cells depends, at least in part, on the expression of HERG. The results reported herein provides an intriguing foundation for a deeper understanding of the mechanism by which cisplatin kills gastric cancer cells, and they indicate that HERG may serve as a potential therapeutic target for cisplatin chemotherapy in human gastric cancer.

\section{Acknowledgements}

This study was supported by Natural Science Foundation of Heilung-kiang Province (no. D2007-69) and the Key Project of Harbin Science and Technology Bureau (nos. 2007AA3CS083 and 2007AA3CS083-1).

\section{References}

1. Jemal A, Bray F, Center MM, Ferlay J, Ward E and Forman D: Global cancer statistics. CA Cancer J Clin 61: 69-90, 2011.

2. Wagner AD, Unverzagt S, Grothe W, Kleber G, Grothey A, Haerting J and Fleig WE: Chemotherapy for advanced gastric cancer. Cochrane Database Syst Rev 17: CD004064, 2010.

3. Zhang LJ, Hao YZ, Hu CS, Ye Y, Xie QP, Thorne RF, Hersey P and Zhang XD: Inhibition of apoptosis facilitates necrosis induced by cisplatin in gastric cancer cells. Anticancer Drugs 19: $159-166,2008$ 
4. Marklund L, Henriksson R and Grankvist K: Cisplatin-induced apoptosis of mesothelioma cells is affected by potassium ion flux modulator amphotericin B and bumetanide. Int J Cancer 93: 577-583, 2001

5. Brickley SG, Revilla V, Cull-Candy SG, Wisden W and Farrant M: Adaptive regulation of neuronal excitability by a voltage-independent potassium conductance. Nature 409: 88-92, 2001.

6. Crociani O, Guasti L, Balzi M, Becchetti A, Wanke E, Olivotto M, Wymore RS and Arcangeli A: Cell cycle-dependent expression of HERG1 and HERG1B isoforms in tumor cells. J Biol Chem 278: 2947-2955, 2003

7. Wulff H, Castle NA and Pardo LA: Voltage-gated potassium channels as therapeutic targets. Nat Rev Drug Discov 8: 982-1001, 2009.

8. Camacho J: Ether à go-go potassium channels and cancer. Cancer Lett 233: 1-9, 2006.

9. Afrasiabi E, Hietamäki M, Viitanen T, Sukumaran P, Bergelin N and Törnquist K: Expression and significance of HERG (KCNH2) potassium channels in the regulation of MDA-MB-435S melanoma cell proliferation and migration. Cell Signal 22: 57-64, 2010.

10. Sanguinetti MC and Tristani-Firouzi M: Herg potassium channels and cardiac arrhythmia. Nature 440: 463-469, 2006.

11. Shao XD, Wu KC, Guo XZ, Xie MJ, Zhang J and Fan DM: Expression and significance of HERG protein in gastric cancer. Cancer Biol Ther 7: 45-50, 2008.

12. Lastraioli E, Guasti L, Crociani O, Polvani S, Hofmann G, Witchel H, Bencini L, Calistri M, Messerini L, Scatizzi M, Moretti R, Wanke E, Olivotto M, Mugnai G and Arcangeli A HERG1 gene and HERG1 protein are overexpressed in colorectal cancers and regulate cell invasion of tumor cells. Cancer Res 64 606-611, 2004

13. Cherubini A, Taddei GL, Crociani O, Paglierani $M$ Buccoliero AM, Fontana L, Noci I, Borri P, Borrani E, Giachi M, Becchetti A, Rosati B, Wanke E, Olivotto M and Arcangeli A: HERG potassium channels are more frequently expressed in human endometrial cancer as compared to non-cancerous endometrium. Br J Cancer 83: 1722-1729, 2000.

14. Masi A,Becchetti A, Restano-Cassulini R,Polvani S, Hofmann G Buccoliero AM, Paglierani M, Pollo B, Taddei GL, Gallina P, Di Lorenzo N, Franceschetti S, Wanke E and Arcangeli A: HERG1 channels are overexpressed in glioblastoma multiforme and modulate VEGF secretion in glioblastoma cell lines. $\mathrm{Br} \mathrm{J}$ Cancer 93: 781-792, 2005.

15. Thomas D, Bloehs R, Koschny R, Ficker E, Sykora J, Kiehn J, Schlömer K, Gierten J, Kathöfer S, Zitron E, Scholz EP, Kiesecker C, Katus HA and Karle CA: Doxazosin induces apoptosis of cells expressing HERG $\mathrm{K}^{+}$channels. Eur J Pharmacol 579: 98-103, 2008

16. Downie BR, Sánchez A, Knötgen H, Contreras-Jurado C, Gymnopoulos M, Weber C, Stühmer W and Pardo LA: Eag1 expression interferes with hypoxia homeostasis and induces angiogenesis in tumors. J Biol Chem 283: 36234-36240, 2008.
17. Dolderer JH, Schuldes H, Bockhorn H, Altmannsberger M, Lambers C, von Zabern D, Jonas D, Schwegler H, Linke R and Schröder UH: HERG1 gene expression as a specific tumor marker in colorectal tissues. Eur J Surg Oncol 36: 72-77, 2010.

18. Asher V, Sowter H, Shaw R, Bali A and Khan R: Eag and HERG potassium channels as novel therapeutic targets in cancer. World J Surg Oncol 8: 113, 2010.

19. Shao XD, Wu KC, Hao ZM, Hong L, Zhang J and Fan DM: The potent inhibitory effects of cisapride, a specific blocker for human ether-a-go-go-related gene (HERG) channel, on gastric cancer cells. Cancer Biol Ther 4: 295-301, 2005.

20. Ding XW, Yang WB, Gao S, Wang W, Li Z, Hu WM, Li JJ and Luo HS: Prognostic significance of herg1 expression in gastric cancer. Dig Dis Sci 55: 1004-1010, 2010.

21. Wang J, Ma Y, Jiang H, Zhu H, Liu L, Sun B, Pan S, Krissansen GW and Sun X: Overexpression of von Hippel-Lindau protein synergizes with doxorubicin to suppress hepatocellular carcinoma in mice. J Hepatol 55: 359-368, 2011.

22. Ma Y, Wang J, Liu L, Zhu H, Chen X, Pan S, Sun X and Jiang H: Genistein potentiates the effect of arsenic trioxide against human hepatocellular carcinoma: role of Akt and nuclear factor- $\kappa \mathrm{B}$. Cancer Lett 301: 75-84, 2011.

23. Zhao W, Zhou SF, Zhang ZP, Xu GP, Li XB and Yan JL: Gambogic acid inhibits the growth of osteosarcoma cells in vitro by inducing apoptosis and cell cycle arrest. Oncol Rep 25: 1289-1295, 2011.

24. Pavet V, Beyrath J, Pardin C, Morizot A, Lechner MC, Briand JP, Wendland M, Maison W, Fournel S, Micheau O, Guichard G and Gronemeyer H: Multivalent DR5 peptides activate the TRAIL death pathway and exert tumoricidal activity. Cancer Res 70: 1101-1110, 2010.

25. Gong JH, Liu XJ, Shang BY, Chen SZ and Zhen YS: HERG K ${ }^{+}$ channel related chemosensitivity to sparfloxacin in colon cancer cells. Oncol Rep 23: 1747-1756, 2010.

26. Spitzner M, Ousingsawat J, Scheidt K, Kunzelmann K and Schreiber R: Voltage-gated $\mathrm{K}^{+}$channels support proliferation of colonic carcinoma cells. FASEB J 21: 35-44, 2007.

27. Roy J, Vantol B, Cowley EA, Blay J and Linsdell P: Pharmacological separation of $\mathrm{hEAG}$ and $\mathrm{hERG} \mathrm{K}^{+}$channel function in the human mammary carcinoma cell line MCF-7. Oncol Rep 19: 1511-1516, 2008

28. Chen SZ, Jiang M and Zhen YS: HERG $\mathrm{K}^{+}$channel expressionrelated chemosensitivity in cancer cells and its modulation by erythromycin. Cancer Chemother Pharmacol 56: 212-220, 2005.

29. Wang H, Zhang Y, Cao L, Han H, Wang J, Yang B, Nattel S and Wang Z: HERG $\mathrm{K}^{+}$channel, a regulator of tumor cell apoptosis and proliferation. Cancer Res 62: 4843-4848, 2002.

30. Han H, Wang J, Zhang Y, Long H, Wang H, Xu D and Wang Z: HERG K channel conductance promotes $\mathrm{H}_{2} \mathrm{O}_{2}$-induced apoptosis in HEK293 cells: cellular mechanisms. Cell Physiol Biochem 14: 121-134, 2004. 